\title{
Le livre au microscope
}

Petites descriptions écologiques à partir de l'Égypte

\section{Christine Jungen}

\section{(2) OpenEdition}

Journals

Édition électronique

URL : https://journals.openedition.org/tc/8643

DOI : $10.4000 /$ tc. 8643

ISSN : 1952-420X

Éditeur

Éditions de l'EHESS

\section{Édition imprimée}

Date de publication : 18 décembre 2017

Pagination : 196-213

ISBN : 978-2-7132-2708-0

ISSN : 0248-6016

\section{Référence électronique}

Christine Jungen, "Le livre au microscope », Techniques \& Culture [En ligne], 68 | 2017, mis en ligne le 18 décembre 2019, consulté le 29 septembre 2022. URL : http://journals.openedition.org/tc/8643 : DOI : https://doi.org/10.4000/tc.8643 


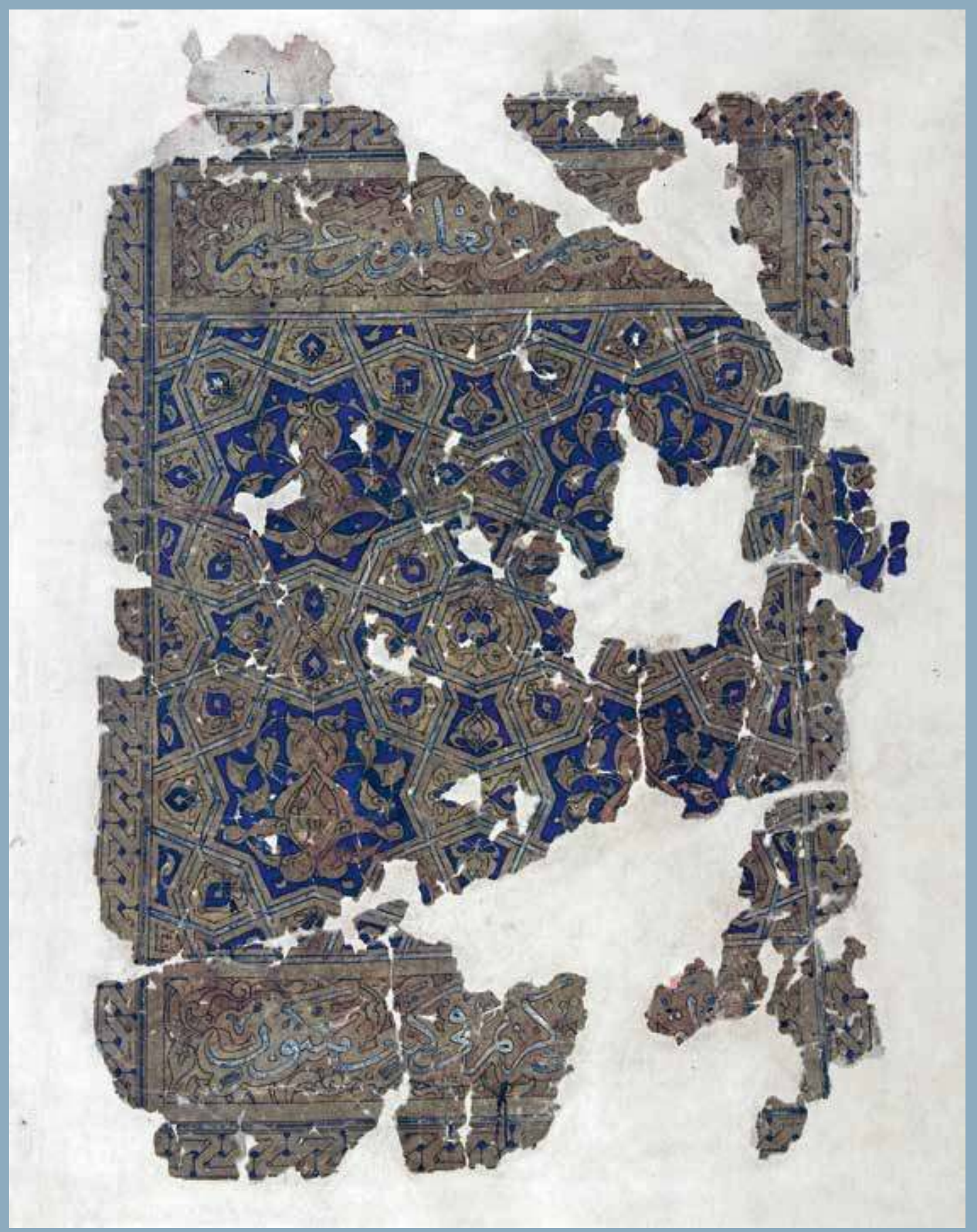




\section{Le livre au microscope Petites descriptions écologiques à partir de l’Égypte}

\section{Texte, codex et stylo-bille}

Le Caire, Bibliothèque nationale, années 1960.

Un vieux savant aveugle d'al-Azhar, la prestigieuse université d'études islamiques, et son élève sont installés dans la section des manuscrits du Dar al Kutub, la «maison des livres» comme est nommée la Bibliothèque nationale d'Égypte. L'élève, un jeune homme, armé d'un stylo-bille, est en train de recopier le manuscrit ancien qu'il a sous les yeux. Il lit le texte à voix haute, sous l'oreille attentive de son aîné, qui commente au fur et à mesure de la lecture. Le lettré approuve parfois d'un geste de la tête ce qu'il entend; plus fréquemment toutefois il intervient, corrigeant le texte au cours de la transcription à l'aide de ce que lui connaît comme étant une version plus « correcte». Son élève suit ses instructions : il modifie le texte lors de sa transcription, et n'hésite pas également à reporter, à l'aide de son stylo-bille, les modifications proposées par son maître directement sur les feuillets du manuscrit original.

L'anecdote est rapportée par l'islamologue Muhsin Mahdi, qui note au passage qu'elle se passe sous les yeux du directeur du département des manuscrits qui pourtant n'interviendra pas, «n'osant protester contre cette pratique (Mahdi 1995: 15). Comme le signale l'auteur, la scène à laquelle il a assisté est révélatrice de la coexistence, et parfois de la tension existant entre deux conceptions du livre manuscrit: celle, issue de la tradition manuscrite de transmission des textes, qui considère le livre manuscrit comme toujours susceptible de tashih, « correction», inscrit dans la tradition de la nuskha, la «copie» ou « version » et sa variance, pour emprunter à Bernard Cerquiglini ${ }^{1}$; et celle, plus récente, du manuscrit comme volume matériel, le codex ${ }^{2}$, à savoir un artefact singulier, doté d'une histoire matérielle, qu'il convient de stabiliser. 
Les livres manuscrits tiennent une place particulière dans l'histoire de l'écrit du monde arabophone: l'imprimerie en caractères arabes ne s'implante véritablement qu'à la fin du xixe siècle, et la production de livres sous forme manuscrite reste vivace jusqu'au début du xxe siècle, avec ses lieux - en particulier les ateliers de copistes - ses praticiens - les scribes, copistes, lettrés - et ses modes de circulation et de transmission - des lectures à haute voix, et des amendements incessants en marge ou dans le corps du texte (Déroche \& Richard 1997). Le nombre de manuscrits en langue arabe est aujourd'hui évalué à plusieurs millions, dont une grande part demande encore à être cataloguée et éditée.

L'économie des manuscrits s'est radicalement transformée depuis le début du xxe siècle. De la tradition de la copie aux pratiques d'édition de manuscrits qui commencent à être mises en œuvre à la toute fin du xix ${ }^{e}$ siècle, le travail sur les manuscrits a, avec la diffusion de l'imprimerie, essentiellement porté sur la production, à l'aide de la comparaison critique des copies manuscrites, de textes édités ${ }^{3}$. L'émergence, à partir des années 1980, d’une codicologie qui s'applique désormais aux manuscrits arabes va solidifier un intérêt pour le manuscrit comme codex, pour le manuscrit conçu donc comme un artefact historique, doté d'un parcours de vie (Déroche 2000). Aujourd'hui, un manuscrit peut être lu comme texte, mais il est aussi saisi -et c'est en particulier le cas des praticiens travaillant dans les collections de manuscrits-dans ses qualités matérielles, qui nécessitent une appréhension extrêmement fine des textures du papier et des cuirs, des encres, des altérations (traces d'humidité, détériorations amenées par les insectes...), bref de toutes les marques et signes qui d'une part servent à identifier un manuscrit précis (dans une famille de copies d'un même texte), et d'autre part permettent de dessiner une biographie du codex. En d'autres termes, le manuscrit arabe s'est, de livre à lire, doublé d'un document historique qui porte dans sa matérialité même les traces d'un passé qu'il s'agit désormais de décrypter en déchiffrant des indices, des plus visibles - les sceaux de propriétés permettant de retracer le parcours social du codex - aux plus fantomatiques - les écritures effacées que l'on peut désormais faire apparaître sur les palimpsestes à l'aide de l'imagerie multispectrale.

Le travail de densification progressive du livre manuscrit - dont la scène de correction au stylo-bille évoquée au début de ce texte montre qu'elle ne s'est pas faite sans heurts- s'est accompagné de nouveaux modes de gestion des documents historiques, en termes à la fois de traitement, de préservation et de restauration. Ceux-ci sont particulièrement prégnants en Égypte, où se multiplient, depuis plus d'une dizaine d'années, programmes de restauration, de catalogage et de numérisation. L'Égypte, un des pays les plus riches en collections de manuscrits arabes anciens - parmi lesquelles en particulier celle du Dar al Kutub, la bibliothèque nationale, qui compte près de soixante mille pièces-, connaît aujourd'hui un foisonnement de projets liés à la pratique des manuscrits : dispositif ambitieux de restauration mis en place dans le cadre de la Bibliothèque d'Alexandrie inaugurée en 2002, programmes patrimoniaux lancés sous l'égide de l'Unesco ou encore de l'Union européenne ${ }^{4}$, sans compter les projets plus spécifiquement liés aux réseaux académiques des spécialistes des manuscrits arabes ${ }^{5}$.

En 2013, l'Institut des manuscrits arabes, fondé en 1946 au Caire, affilié à l'Organisation arabe pour l'éducation, la culture et les sciences (elle-même dépendant de la Ligue arabe), et 
spécialisé dans le catalogage, l'édition et la préservation des manuscrits arabes, annonce la tenue de la première «Journée du manuscrit arabe». Pour son édition inaugurale au Caire organisée en collaboration avec la Bibliothèque d'Alexandrie, la Journée, destinée à faire découvrir au grand public les richesses des manuscrits, propose conférences, exposition, films, ateliers et jeux. L'annonce officielle de l'événement est emphatique: «Le manuscrit arabe est un être historique ( $k a^{\prime} i n$ tarikhi) qui défie la mort, et qui, après plus de quinze siècles, continue à vivre. Il emmagasine des mondes de la pensée, de la science, de la connaissance, de l'art et des techniques, qui demandent donc une approche fondée sur le mélange extraordinaire des plaisirs de la découverte, de la stimulation et de la surprise.»

Comment est produit, aujourd'hui, un «manuscrit arabe ${ }^{6}$ » encapsulant des «mondes»? Si l'on prend la formule au mot dans une version pragmatique, l'examen des pratiques entourant les collections de manuscrits révèle des mondes qui sont tout d'abord ceux des dispositifs matériels et des environnements relationnels dans lesquels sont déclinées et connectées des échelles de plus en plus minimales de prises sur le manuscrit. Cet examen livre également, ce faisant, un panorama où s'enchevêtrent les champs du turath (patrimoine) et ceux du 'ilm (savoir, science), se mêlent réseaux locaux et internationaux de coopération et de concurrence, où gravitent chimistes et codicologues, savants et techniciens, conservateurs et lettrés. Par quels assemblages sont captés les signaux de l'histoire? Comment sont façonnés, efficacement, dans ces lieux, des «êtres historiques »? À travers une série d'incursions dans des dispositifs de préservation et de conservation, ce sont ces écologies du livre manuscrit dont je propose ici un récit.

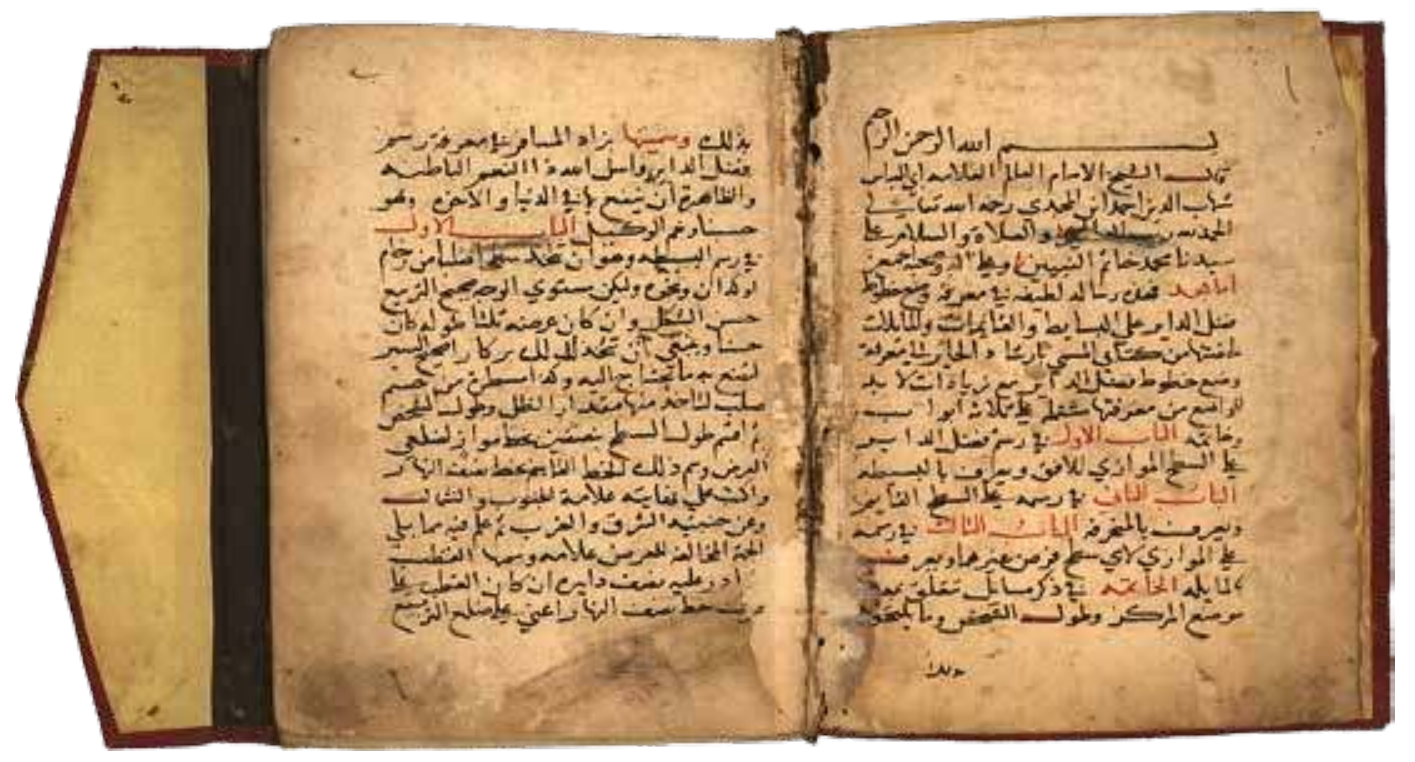

1. Le Guide du voyageur sur le tracé du cercle de projection par Ibn al Majdi, manuscrit achevé vers 1637. 


\section{Pest Control, gaz et taux de pH : du rayonnage à la paillasse}

Au début des années quatre-vingt-dix, un archiviste français est chargé par l'Unesco d'évaluer l'état des archives et documents historiques en Égypte. Si le panorama qu'il dresse dans son rapport est celui de fonds dans des états très contrastés, il dénote des services souvent « déficients », voire «archaïques » : à la Bibliothèque nationale établie au Caire dont dépend la section des Archives nationales, le missionné relève en particulier le

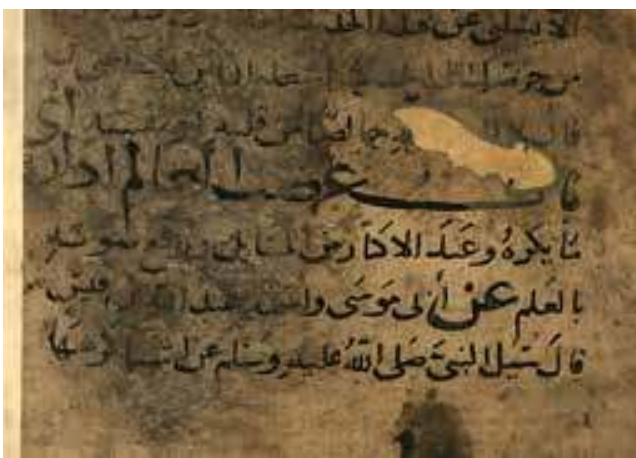

2. Détails de manuscrits Détériorations et réparations manuelles telles qu'elles étaient effectuées avant le grand chantier de rénovation des équipements de restauration du Dar al Kutub.

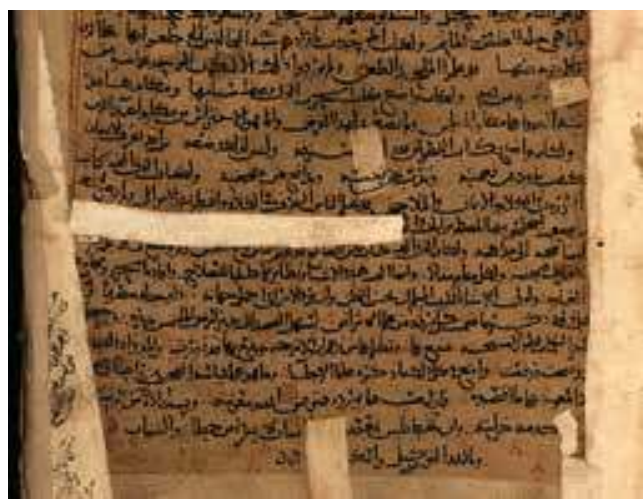
bâtiment en béton datant de la fin des années soixante, peu adapté à la conservation documentaire, et ses fenêtres grandes ouvertes sur la ville - connue pour ses poussières tenaces ramenées par le vent du désert environnant, sans compter l'importante pollution urbaine - en raison d'une climatisation inopérante. Le personnel, s'il est qualifié et maîtrise parfaitement la restauration manuelle, manque en revanche cruellement de moyens, souligne l'archiviste, et notamment du matériel adapté pour traiter le papier ancien. La situation décrite à Alexandrie, deuxième ville du pays, est encore plus morne: des fonds d'archives et des milliers de manuscrits anciens attendent, «dans un état critique», entassés dans la cage d'escalier du Palais du Gouverneur, leur éventuel transfert dans les locaux, alors provisoires, de la Bibliothèque d'Alexandrie (qui sera ouverte en 2002), ou encore des masses de documents qui sont empilés « en vrac, dans un état dramatique» sous le stadium de la ville. Le rapport préconise, dans ses conclusions, un équipement complet en matériel de restauration du Dar al Kutub, et l'organisation immédiate de stages à l'étranger pour le personnel dans « des services pourvus de moyens équivalents (c'est-à-dire minimum pour l'instant)».

Vu de France, qui plus est par un inspecteur général des Archives de France, le tableau doit, effectivement, paraître sombre. L'Égypte est loin, alors, des moyens techniques de conservation français où mesure du taux d'acidité du papier, des taux d'humidité de l'air ou encore identification précise des bactéries ou champignons responsables des moisissures font partie du travail presque routinier de la conservation des fonds documentaires.

Pourtant, ce n'est qu'au terme d'un mouvement, qui fut très progressif, d'introduction de techniques scientifiques dans les collections patrimoniales et historiques que les dispositifs chimiques, bactériologiques, et plus largement environnementaux ont investi les collections documentaires. Dans la sinueuse histoire de l'introduction des techniques de laboratoire dans les collections historiques, qui épouse, avec des détours et des bifurcations, celle des sciences, on peut repérer quelques dates et figures: partons de la plus connue dans l'histoire de l'observation scientifique, celle du jeune Robert Hooke, membre de la Royal Society et ancien assistant de John Wilkins et de William Boyle, qui publie, en 1665, 
Micrographia, un traité illustré sur le monde microscopique. Parmi ses descriptions de l'univers qui émerge "vu du microscope », qui constitueront un basculement décisif dans la représentation et la perception collectives du monde (Shapin 1998), le récit détaillé d'une moisissure qui a élu domicile dans la couverture en cuir d'un de ses livres, ainsi que du book-worm, le «ver des livres», à propos duquel il note: "This Animal probably feeds upon the Paper and covers of Books, and perforates in them several small round holes, finding, perhaps, a convenient nourishment in those hulks of Hemp and Flax, which have pass'd through so many scourings, washings, dressings and dryings, as the parts of old Paper must necessarily have suffer'd; the digestive faculty, it seems, of these little creatures being able yet further to work upon those stubborn parts, and reduce them into another form.» (Hooke 1665: 209-210).

Sa notice sur le «ver des livres » est largement citée et critiquée dans un pamphlet qui deviendra un best-seller, publié en 1880 par le bibliophile William Blades sous le titre The Enemies of Books. Blades, qui a joué un rôle actif dans la fondation en 1877 de la Library Association, l'ancêtre de l'association professionnelle britannique des bibliothécaires, y fait un plaidoyer passionné pour la préservation des livres anciens, et lance une charge aussi violente que sarcastique contre leurs agresseurs humains et non humains. Parmi ceux-ci, il énumère en vrac les bibliothécaires négligents, les collectionneurs «biblioclastes», les serviteurs trop empressés à nettoyer les livres, les femmes et les enfants, mais aussi la chaleur, les larves d'insectes (à propos desquelles Blades raconte avec humour avoir réussi à faire vivre pendant près de quatorze mois une larve venue d'Athènes et «nourrie à la tradition hébraïque »), les gaz, les fumées et les poussières. Le succès du pamphlet, le premier à décliner une véritable écologie du livre ancien qui s’accorde aux préoccupations environnementales et hygiénistes émergentes de l'époque, est tel qu'il sera réédité plusieurs années durant.

C'est toutefois en France, dès les années 1840, qu'ont été publiées les premières expérimentations directement influencées par l'avancement des connaissances en physique et en chimie. Alfred Bonnardot, autre bibliophile et passionné d'histoire parisienne, décide, face aux «renseignements en général assez ineptes » proposés par les notes existantes sur le sujet, d'appliquer à la restauration du papier les notions acquises dans sa jeunesse dans les cours de physique et chimie de Thénard et Gay-Lussac. De ses «expérimentations » menées pendant six ans et de ses lectures des «ouvrages de chimie les plus modernes» dont il s'inspire pour trouver les procédés les plus adéquats, il publie en 1846 un livre, Essai sur la restauration des estampes anciennes et des livres rares, ou Traité sur les meilleurs procédés à suivre pour réparer, détacher, décolorier et conserver les gravures, estampes et livres. Il y détaille notamment les solvants utilisables pour nettoyer les différents types de tache, les méthodes de blanchissement du papier et ses risques d'acidification, et souligne la nécessité d'une hygiène documentaire, remarquant avec pessimisme à l'égard des générations futures: «Malgré le goût actuel du siècle pour la conservation de tous les genres de monuments (supposé que ce goût ne passe pas comme une mode), je doute néanmoins que nos descendants, dans deux siècles d'ici, puissent s'exempter de restaurer toutes les gravures ou lithographies sorties de nos presses depuis 1825. À partir environ de cette époque, nos papiers cotonneux, blanchis au chlore et fabriqués à l'aune, n'offrent en général aucune chance de durée.» (Bonnardot 1846: 71-72). 


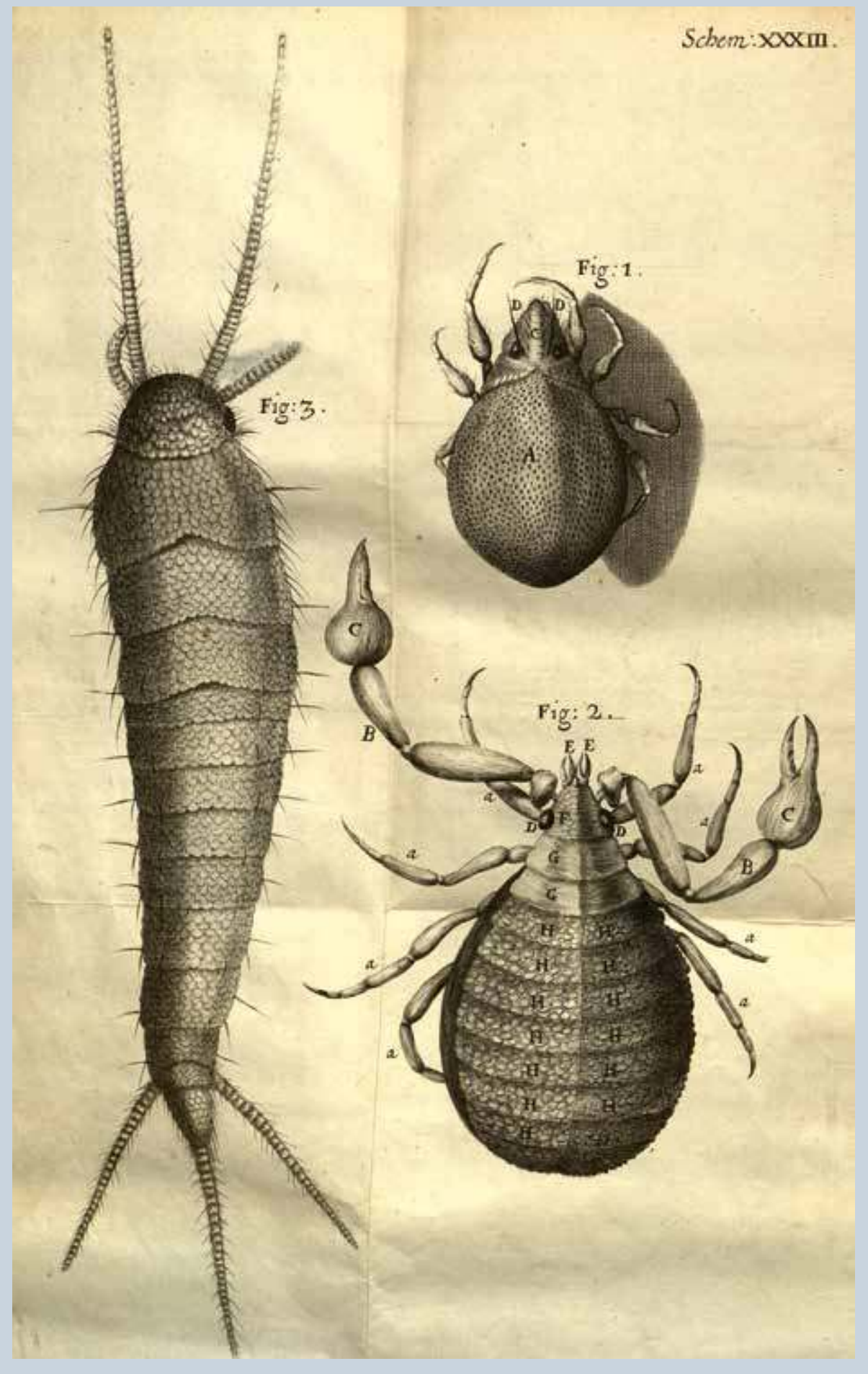

3. Mite, pou et un ver des livres, puis ci-contre des moisissures, vus par Robert Hooke

Les moisissures, dessinées à partir de celles qu'il découvre sur la couverture en cuir d'un petit livre, sont en particulier une source d'émerveillement pour Hooke qui en commente longuement les «très jolies formes » et la «constitution tendre». 


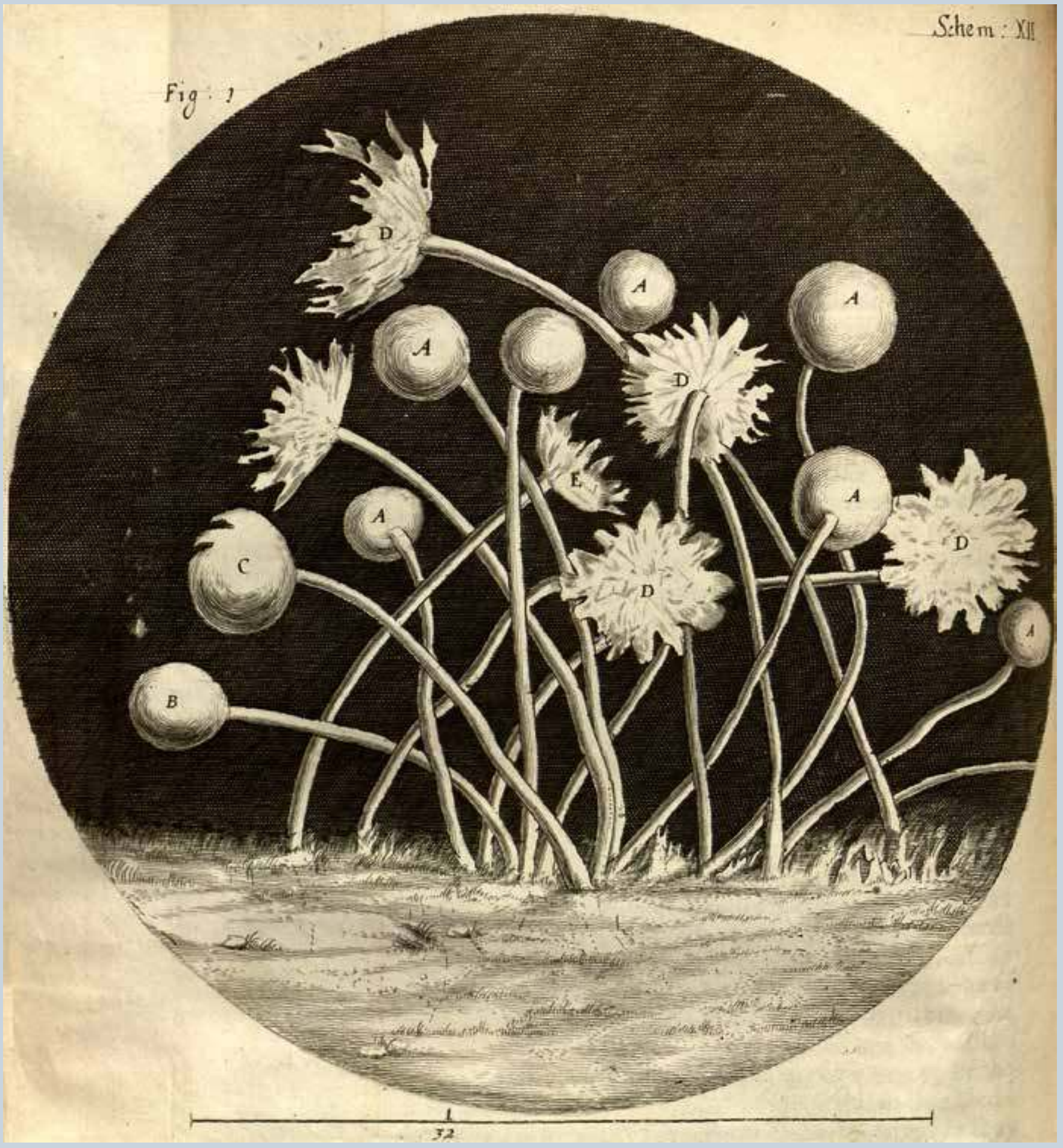



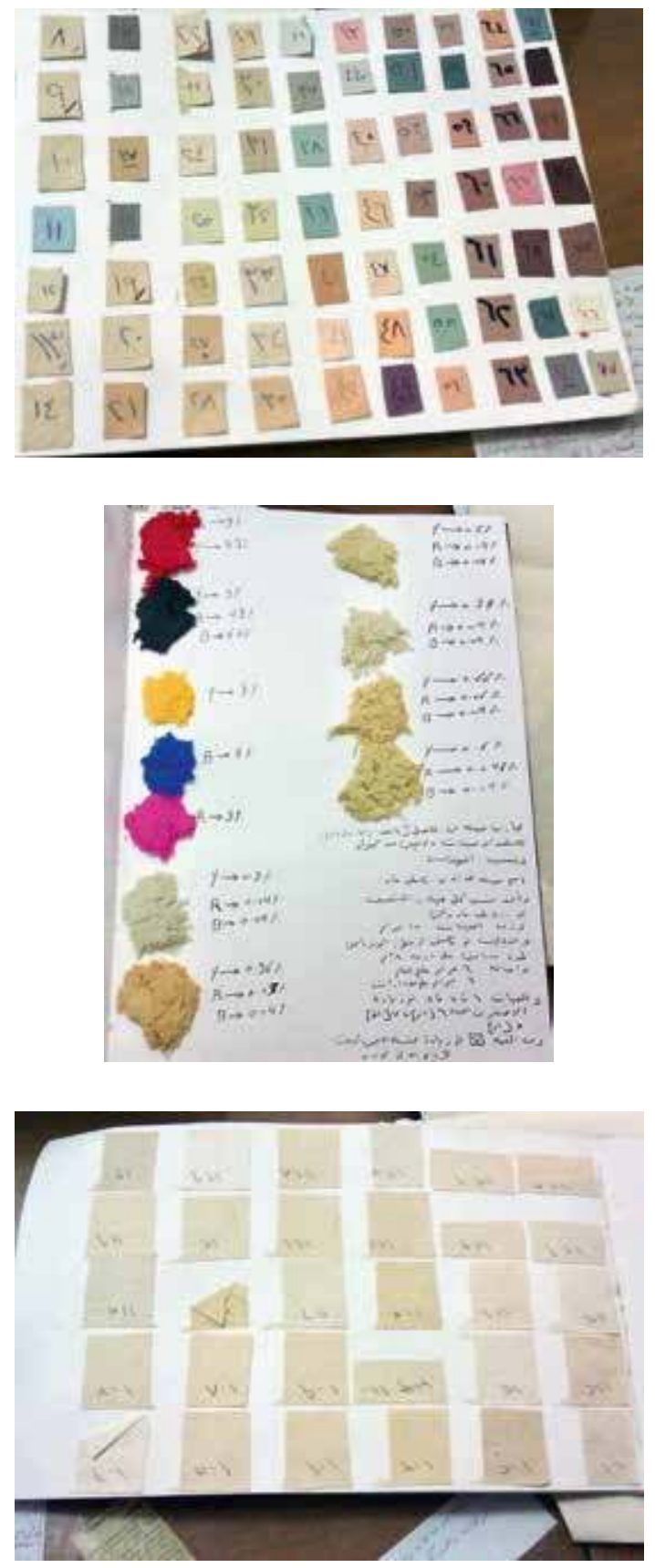

Dosage des couleurs et échantillons de teinte au Dar al Kutub
Le «goût pour la conservation» ne passe pas. En 1907, le DrFernand Berlioz, fondateur de la chaire de bactériologie de l'École de médecine, rédige un mémoire sur la Désinfection des livres fermés, dans lequel il préconise de traiter au gaz désinfectant les collections touchées par les moisissures de champignons et bactéries. En Italie dans les années 1930, le paléographe et bibliothécaire Alfonso Gallo expérimente la collaboration entre restaurateurs du livre, chimistes et biologistes. Sous sa direction est fondé, en 1938, l'Institut de pathologie du livre, premier institut à pleinement se consacrer à la préservation des volumes matériels.

Revenons au Caire contemporain, une bonne vingtaine d'années après le passage de notre archiviste français. Le Dar al Kutub est aujourd'hui engagé dans une vaste restauration de ses fonds et collections de documents et livres anciens. Des centaines d'employés sont affairés, qui dans la section des Archives nationales, qui dans la section des manuscrits, à la restauration manuelle des documents. Ils sont entourés de scalpels, colles et papiers japon, le papier communément utilisé en restauration, et renforcent, réparent ou recomposent inlassablement des feuillets troués par les larves d'insectes, déchirés ou émiettés. Dans la section des manuscrits, cinq restaurateurs entourent un immense exemplaire du Coran, richement illuminé: après en avoir restauré les feuillets un par un, ils sont en train de remonter les pièces du volume, et discutent de la qualité du rendu. La brillance des couleurs qu'ils ont réussi à obtenir après un long travail de gommage et de réparation les émerveille, et ils palpent, satisfaits de leur travail, les pages du manuscrit ainsi restauré. Dans la salle d'à côté, des techniciens fabriquent, en mesurant les rapports de jaune, rouge et bleu, les différentes couleurs de cellulose utilisée pour restaurer le papier: des séries d'échantillons sont à la disposition des restaurateurs, qui ont plus d'une centaine de teintes, dûment étiquetées (du blanc au jaune foncé, voire marron clair, en passant par différentes teintes de rose), à leur disposition pour choisir celle qui sera «au plus proche» du papier d'origine. Un peu plus loin, des bacs de solution aqueuse dans lesquels sont plongés les feuillets afin de désacidification.

Dans une arrière-salle, un homme, seul, est assis sur une chaise. Il surveille patiemment des boîtes translucides accrochées aux murs de la salle, dans lesquelles il cultive, littéralement, des larves d'insectes. Dans chaque boîte, des dizaines de larves 
s'agitent autour de feuillets et miettes de papier sous son œil attentif. Son travail est de surveiller le cycle de vie - et notamment de reproduction - des insectes bibliophages, au premier rang desquels les vrillettes et les poissons d'argent qui infestent les collections. Un étage plus haut, une autre forme de saisie des vies microscopiques est opérée au laboratoire de microbiologie. Trois microbiologistes sont penchés sur un écran d'ordinateur, examinant avec attention une souche. Ici, on parle vrillettes, mais aussi champignons, moisissures, gaz et stérilisation, on se déplace entre paillasses couvertes de cultures prélevées sur les manuscrits, le microscope et les écrans d'ordinateur, on relie, par le biais des changements d'échelles et des opérations successives de captation et de visualisation, les altérations visibles (les taches et les trous que nettoient ou réparent ensuite les restaurateurs) aux entités microscopiques qui en sont la cause.

Les magasins dans lesquels sont remisés les manuscrits une fois restaurés sont attentivement contrôlés. Les microbiologistes opèrent régulièrement des prélèvements afin de vérifier l'absence de développement de moisissures, traquent inlassablement toute apparition de nuisibles susceptibles de s'attaquer aux livres. Taux d'humidité et température, mais aussi des gaz d'échappement, font l'objet d'une surveillance rigoureuse par le laboratoire de pollution, à l'aide des capteurs qui permettent de contrôler la qualité de l'air.

De livre à lire, feuilleter, corriger, recopier, le manuscrit est devenu une matière enserrée fermement dans des dispositifs de traitement, de captation et de stabilisation qui mettent en branle des collectifs de plus en plus complexes. De simple réparation manuelle, le traitement du manuscrit s'est transformé en soin environnemental, routinier et fragmenté - chaque spécialiste dans la chaîne de travail étant concentré sur son segment d'action dans la chaîne opératoire -, et découpé dans des échelles de plus en plus fines. Dans la restauration du coran viennent s'imbriquer séparément problèmes de pollution urbaine, cercles chromatiques, souches bactériennes. Dans le geste de préservation se déploient désormais différents mondes que le codex emporte avec lui: suivre le développement des larves de vrillette, cultiver des souches bactériennes ou encore mesurer le taux d'acidité d'un papier sont aussi, en élargissant les degrés d'intervention sur le codex, des opérations de densification du manuscrit, qui le font, littéralement, «travailler » et «vivre» à différentes échelles.

La mise en œuvre des techniques de sianah (préservation) et de restauration (tarmim) suit, dans son fond et dans ses formes, les préconisations élaborées dans les organisations internationales qui parrainent de manière directe ou indirecte 7 , le travail effectué au Dar al Kutub: Unesco, Ifla (International Federation of Library Association), Icom (International Council of Museums), ou encore Iccrom (International Centre for the Study of the Preservation and Restoration of Cultural Property) publient inlassablement rapports et recommandations, organisent tour à tour conférences, formations et ateliers sur le patrimoine et sa préservation. Lancien bâtiment, où avait été établi le Dar al Kutub en 1899 avant de déménager dans les nouveaux bâtiments construits à cet effet, a été restauré et réaménagé en un musée des manuscrits et une salle de lecture des microfilms. L'atelier de restauration de la Bibliothèque est désormais découpé en Maintenance Unit, Environment Control Unit, Pest Lab et autres Chemical Lab qui opèrent, parfois en se bousculant, sur les différentes échelles des manuscrits. 


\section{Quand le papier s'épaissit: capter les «bons " signaux de l'histoire}

Le développement ces quinze dernières années de techniques de traitement des manuscrits résulte de la circulation de plus en plus rapide de savoir-faire, entre pays comme entre disciplines, doublés d'enjeux qui sont aussi patrimoniaux que politiques. De ce point de vue, la rénovation de ses fonds entreprise par le Dar el Kutub fait aussi suite à l'ouverture en fanfare, en 2002, de la nouvelle Bibliothèque d'Alexandrie sous l'égide de l'Unesco. Projet monumental, la Bibliotheca Alexandrina a été conçue avec une double ambition: réaffirmer sur le monde arabe la prééminence culturelle que l'Égypte revendique historiquement en créant un grand lieu de savoir qui s'inscrit directement dans la filiation de l'ancienne - et mythique, pour la culture européenne et plus largement occidentale - Bibliothèque d'Alexandrie. Dotée à son ouverture des machines les plus modernes grâce à des dons et des financements extérieurs, résolument ouverte sur les institutions et réseaux internationaux et en particulier de la Méditerranée septentrionale, la Bibliotheca Alexandrina se place, dans son ambition à constituer un nouveau pôle régional du savoir, directement en concurrence avec le Dar al Kutub.

Le Centre des manuscrits de la Bibliothèque d'Alexandrie a reçu en 2002 un financement italien grâce auquel il a pu non seulement acquérir des machines dernier cri -une machine de colmatage automatique en particulier - nécessaires à la restauration mécanique, mais aussi envoyer des restaurateurs en formation à Rome. Le Centre des manuscrits est créé sous l'égide de Youssef Ziedan, érudit, romancier, islamologue et intellectuel, qui dirige l'institution jusqu'en 2012. Féru de codicologie, il est aussi grand amateur de catalogage, qu'il considère comme une pratique centrale de l'étude des manuscrits; il publie à ce titre de nombreux catalogues de manuscrits, parmi lesquels en particulier le catalogue en plusieurs volumes de la bibliothèque municipale d'Alexandrie, dont la collection de manuscrits a été transférée au Centre.

Le Centre compte un laboratoire de chimie et de microbiologie, un atelier de restauration manuelle, un atelier de microfilmage, un atelier de numérisation sans oublier la section de catalogage. Le manuscrit à restaurer, à l'instar des trajectoires suivies dans le Dar al Kutub, emprunte un parcours dûment encadré: il est tout d'abord examiné par le restaurateur, qui décompose progressivement le manuscrit au sens littéral comme au figuré: il démembre la couverture, défait les cahiers, sépare les feuillets. Il enlève les scotchs, bandes ou autres réparations existantes. Chaque feuille est examinée attentivement, à charge du restaurateur de repérer et de noter les traces de détériorations visibles à l'œeil nu (galeries creusées par les insectes dans les pages, détériorations des bordures, taches d'humidité, altérations causées par une encre ferrogallique ${ }^{8}$...) et d'effectuer une première identification sommaire des différents composants: matière de la couverture, type d'encre, nature de papier. Les différents éléments du manuscrit, dispersés dans des pochettes en plastique, sont ensuite transférés au laboratoire dans lequel les tests s'affinent: $\mathrm{pH}$ des feuillets, indices d'infection biologique ou bactérienne, prélèvements et analyses des différents éléments organiques du codex. Le manuscrit est ensuite traité selon les besoins et les conclusions de l'analyse, avant d'être renvoyé à la restauration manuelle où le codex va être progressivement reconstitué. 
La restauration demande toucher et dextérité: "On devrait porter des gants, mais j'ai besoin de "sentir" (yhiss) le papier», commente une restauratrice. Cela exige également patience et minutie: les manuscrits qui arrivent dans le centre sont souvent dans un état dégradé, à l'instar de la collection de la bibliothèque municipale de la ville qui a été entièrement restaurée par le centre. Sur chaque page, le colmatage des petits trous laissés par les larves et des craquelures et déchirures, plus ou moins importantes, dont souffrent les bordures peut prendre plusieurs jours. Les restaurateurs, écouteurs reliés à leurs portables vissés sur les oreilles, grattent, aplatissent, rebouchent, réparent, collent, pressent, recousent et recomposent les éléments disparates et fragmentés dont la composition finale doit aboutir au «manuscrit arabe». "Sentir le papier» est affaire de tactilité et de perception, qui vont influer sur des choix en apparence mineurs mais en réalité cruciaux: le papier est-il «faible» (dha'if), ce qui se mesure en le frottant légèrement entre les doigts pour juger de sa minceur et de sa texture, en le pliant tout aussi légèrement pour jauger sa souplesse, et doit-il donc être renforcé? Les trous occasionnés par les insectes ou une encre sont-ils suffisamment grands pour être colmatés un à un, ou faut-il encoller toute la page, ce qui en modifiera la texture mais lui redonnera de l'épaisseur? La couleur du papier choisie pour les colmatages estelle suffisamment proche du papier original pour ne pas dénaturer l'appréhension globale de la page, tout en restant suffisamment visible pour indiquer l'existence de la restauration?

Ce qui est finalement en jeu, dans la logique de soin et de réparation qui régit l'ensemble de la chaîne de restauration, est la sélection, dans l'épaisseur historique proposée, des caractéristiques considérées comme les plus pertinentes, et l'élimination de celles considérées comme "étrangères» au codex. Cette sélection traite le manuscrit un peu à la manière d'un

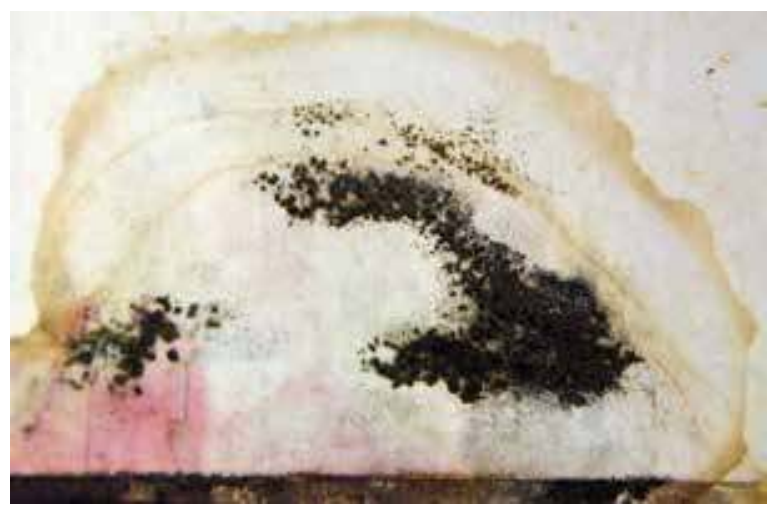

5. Moisissure sur un bord de page

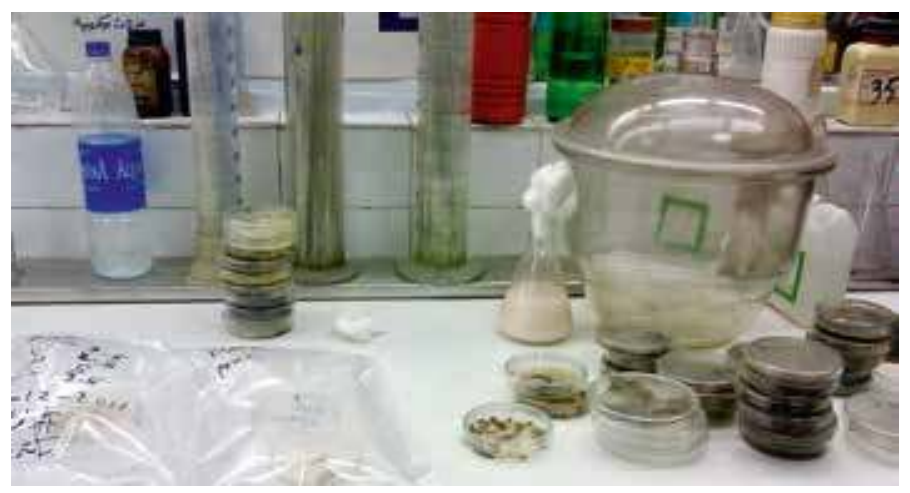

6. De la paillasse à l'ordinateur. Travail de visualisation des souches microbiologiques qui affectent les manuscrits au Dar al Kutub

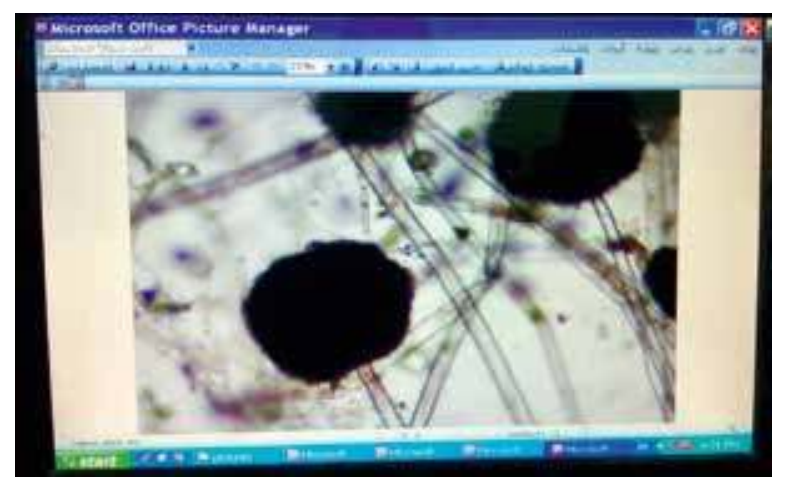




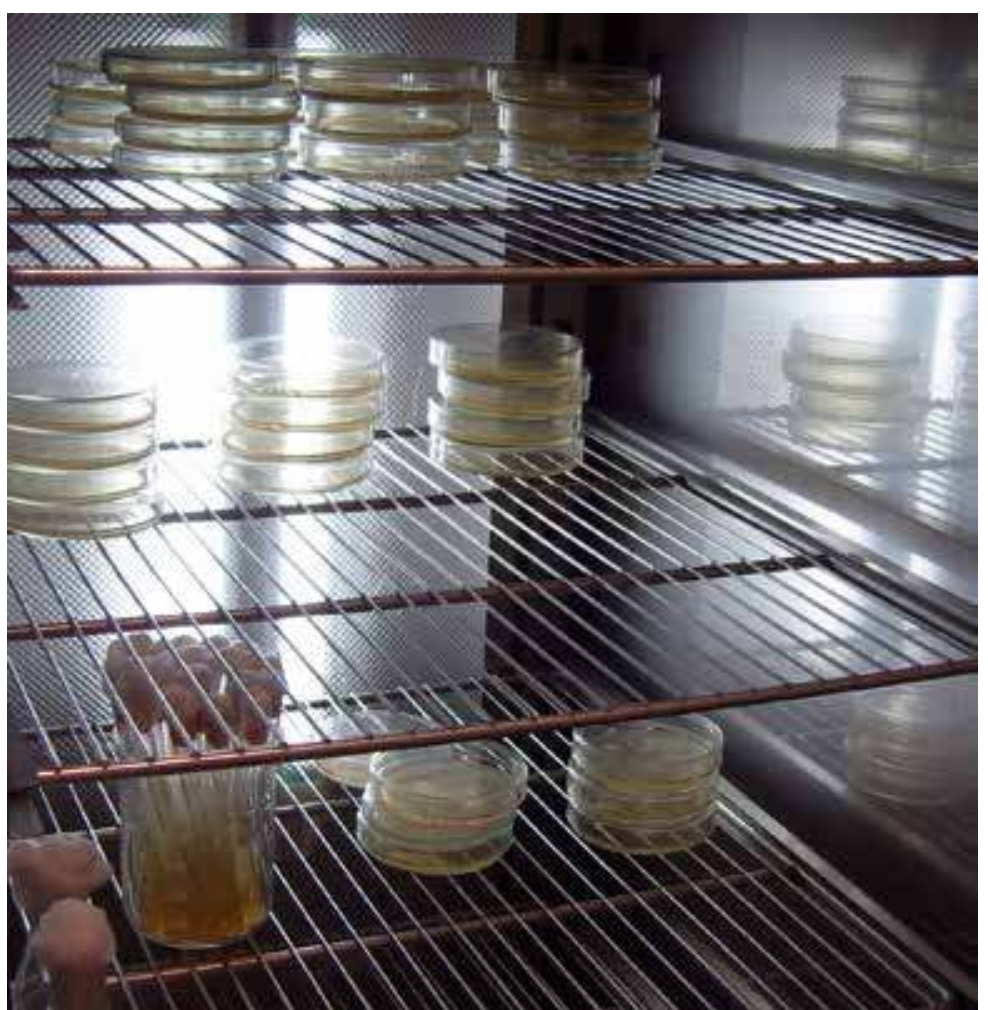

De la culture

Bibliotheca Alexandrina site historique, à densité haute, duquel il faut écarter méticuleusement les «bruits résiduels » - l'ensemble des éléments étrangers à l'époque que le site a pour but de saisir - pour mieux capter et forcir le signal, parfois faible, du codex d'origine: élimination, d'une part, de tout ce qui, après examen, est rejeté dans le domaine des altérations et des effets indésirables (rangés dans le champ du athar, «trace», «vestige »), de ce qui est identifié comme intrus, de la trace des insectes ou moisissures aux raccommodages de fortune effectués un jour par un apprenti restaurateur; consolidation et reconstitution, d'autre part, de ce qui est trié et rangé dans les composantes de l'intégrité historique du document d'origine.

Celle-ci est centrée en premier lieu autour de la lisibilité et de la stabilité du manuscrit. Si Youssef Ziedan, grand admirateur des travaux pionniers de François Déroche dans le domaine de la codicologie arabe, promeut une conception du manuscrit dans ses propriétés matérielles aussi bien que textuelles, c'est bien un codex facilement saisissable dans ses propriétés visuelles de «manuscrit arabe», et surtout

robuste que vise à façonner le centre. Plus encore, ce sont des collections de livres en bonne et due forme, trouvant leur place naturelle dans l'ordre des livres de la bibliothèque du centre, qui sont produites par la chaîne de restauration.

Youssef Ziedan est démis de ses fonctions en mai $20122^{9}$. Un nouveau directeur est nommé, et le musée des manuscrits qui dépendait du Centre prend le pas administrativement sur les activités de catalogage et de recherches; un tournant muséal s'opère avec une réorientation de la politique de préservation: réversibilité des opérations de restauration et contrôle de l'environnement sont désormais les principes directeurs du traitement des manuscrits. En 2015, le musée des manuscrits, qui se targue de suivre les «normes internationales des institutions de restauration mondiales », reçoit le prix de la branche égyptienne de l'Icom (Conseil international des musées) du meilleur laboratoire de restauration en Égypte, fournit son expertise pour la restauration des manuscrits de l'université al Azhar, et commence à traiter pour restauration plusieurs collections extérieures, de la collection des manuscrits du Patriarcat grec orthodoxe à Alexandrie à celle du Diwan royal de l'émirat du Koweït. 
Achevons notre parcours par un détour par le monastère de Sainte-Catherine. Situé dans le Sinaï, le monastère détient une riche collection, une des plus anciennes connues, de manuscrits de langue copte, arabe, hébraïque ou encore syriaque et araméenne. Le monastère a déjà travaillé avec le Centre des manuscrits de la Bibliothèque d'Alexandrie - qui a notamment achevé, en 2008, la numérisation d'une première série de ses manuscrits rares. Mais le père Justin, le responsable de la bibliothèque, engagé dans la numérisation de l'ensemble des collections, bute sur la collection de palimpsestes, dont il n'arrive pas à rendre les écritures lisibles. En quête d'experts susceptibles de trouver une solution technique, il rencontre aux États-Unis un ancien spécialiste de surveillance et d'imagerie satellitaire. Michael Toth, comme il le raconte au Washington Post, s'est converti de la conception de satellites espions à l'étude des manuscrits anciens à la suite de la lecture d'un article: celui-ci relatait les difficultés rencontrées par les spécialistes face à un palimpseste contenant une copie datée du $x^{e}$ siècle d'un texte d'Archimède recouvert d'une deuxième couche d'écriture. Toth a l'idée d'appliquer les techniques de pointe d'espionnage au manuscrit, et contacte le Walter Arts Museum de Baltimore où est conservé le palimpseste ${ }^{10}$. Au sein de l'équipe qui travaille sur le palimpseste, il développe alors des méthodes de photographie de plus en plus pointues des textes à l'aide de filtres améliorant la lisibilité du texte perdu. À la suite de sa rencontre avec le père Justin, le Sinai Palimpsest Project est monté sous la direction du père en collaboration avec des chercheurs américains et européens. Dans le cadre du projet, les techniques d'imagerie multispectre et le développement de machines conçues spécialement pour scanner les manuscrits à différents angles de vue construisent désormais une prise de loin: celle de la caméra qui photographie à haute résolution, à près d'un mètre de hauteur, les portions de feuillets. Vient ensuite la manipulation numérique de la matérialité du manuscrit. L'obtention d'une image satisfaisante repose ainsi aussi bien sur l'ajustement des filtres pour obtenir des variations d'onde à la «bonne longueur» susceptibles de discriminer les encres, les moisissures, le papier, soit toutes les matières composant le manuscrit, que sur les algorithmes qui recombinent les images ainsi obtenues et, surtout, reconnaissent ces différentes « classes » de matière pour recomposer les pixels souvent manquants: il a ainsi fallu, racontent les chercheurs ayant expérimenté l'imagerie multispectrale sur le palimpseste d'Archimède, «"entraîner" l'algorithme à reconnaître le motif de la brillance du pixel rapportée à la longueur d'onde [de manière à ce que] l'ordinateur détermine la probabilité de l'appartenance de chaque pixel à une classe [de composant]» (Easton \& Noel 2004: 43). Dans l'imagerie multispectrale, c'est désormais à l'écran, et à bonne distance du manuscrit, que s'exercent les opérations qui visent à isoler et (re)composer un nouveau signal historique dans le site physique du livre.

Les spécialistes d'histoire de l'art s'accordent sur la révolution dans la discipline qu'a constituée l'introduction de nouvelles techniques - du microscope à la réflectographie infrarougedans l'appréhension des œuvres d'art. Leur impact est particulièrement prégnant pour les tableaux, dont les strates saisies de manière de plus en plus en fine et de plus en plus en profondeur ont fait advenir des histoires et des interprétations alternatives, et bousculé tour à tour les notions d'œuvre, d'auteur, d'original et d'authenticité ${ }^{11}$. Vu d'Égypte, la question n'est pas tant 


\section{L'ordre des livres}

Rayonnages sous verre - salle de lecture des manuscrits, Bibliotheca Alexandrina -, assortis du thermo-hygrographe qui enregistre la température et l'humidité relative de l'air.

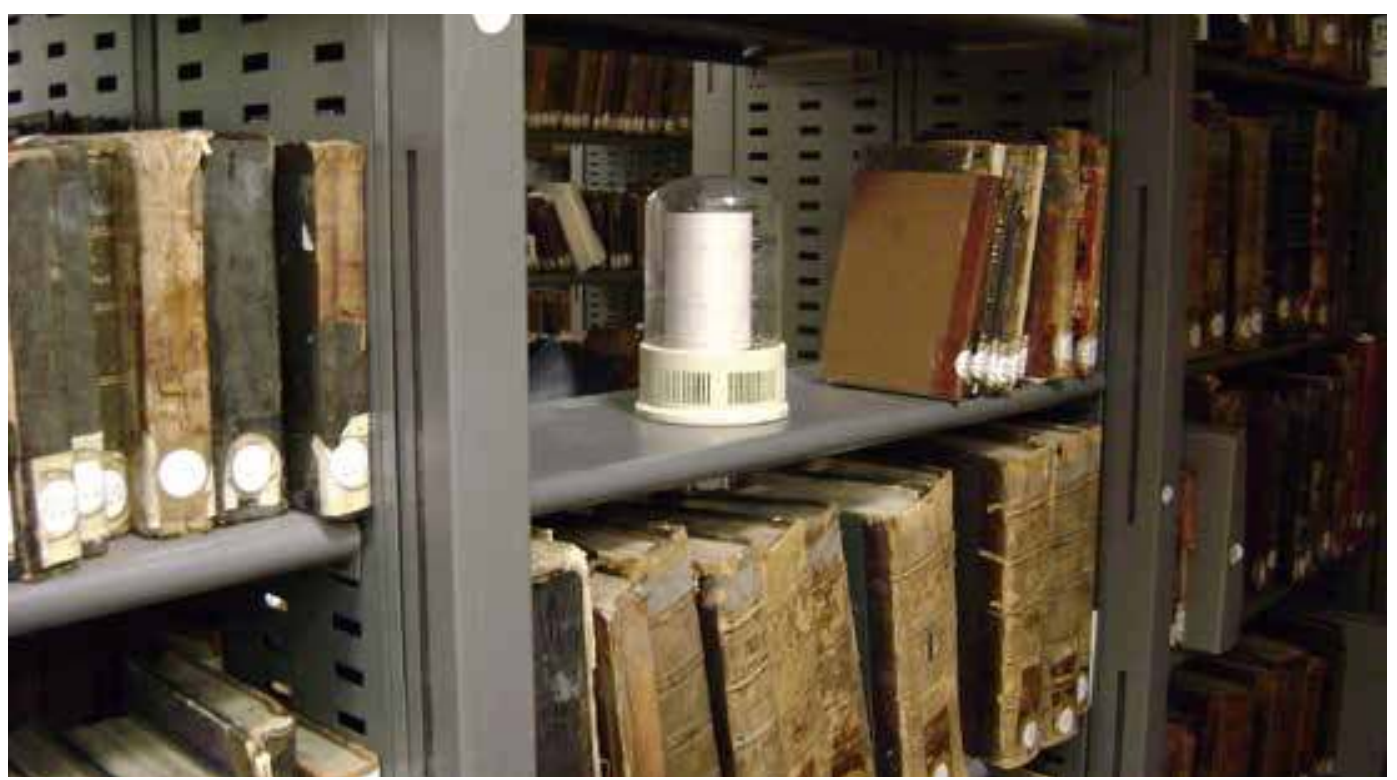

celle, au fond assez inopérante, d'authenticité ou d'original, mais de faire cohabiter des régimes alternatifs et parfois conflictuels de saisie des manuscrits. Ceux-ci opèrent dans des cadres contraints qui sont, en premier lieu, règles à suivre, normes à respecter, coûts à gérer, et peutêtre plus encore, position à tenir ou à imposer sur la scène locale, régionale et internationale. L'intégration des institutions égyptiennes dans les circuits de restauration internationaux, et le passage du manuscrit du rayon de bibliothèque au microscope de laboratoire qui en a résulté, sont, de ce point de vue, moins des moments et des lieux de basculements épistémologiques que de négociations, dans et autour du corps du livre manuscrit, des collectifs à mobiliser et des signaux historiques à sélectionner: ce sont bien, au fond, les possibilités simultanées et différentielles du manuscrit arabe comme «être historique» que décline le travail de densification progressive du livre manuscrit.

Le «manuscrit arabe » constitue sans doute aujourd'hui, dans cette perspective, un site par excellence d'expérimentation textuel, mais aussi matériel de la variance, voire de la version telle que la définit Vinciane Despret: à savoir une proposition qui «renvoie, au-delà d'ellemême, à l'existence d'une autre version » (Despret 2001 : 38). Les déclinaisons et diffractions entre le stylo-bille et l'imagerie multispectrale, les savoirs et les sciences, le patrimoine et l'histoire, les régimes érudits et les régimes muséaux, sont toutes contenues dans les épaisseurs du manuscrit. Les différentes versions qui s'appuient sur ces épaisseurs et strates s'inscrivent, pour suivre à nouveau Despret, « dans le temps d'une histoire, qu'elles prolongent, transforment, retournent ou réarticulent » (ibid. : 44). En ce sens, les manuscrits égyptiens racontent, aussi, les circuits que ces versions créent et les courroies de transmission sur lesquelles elles 

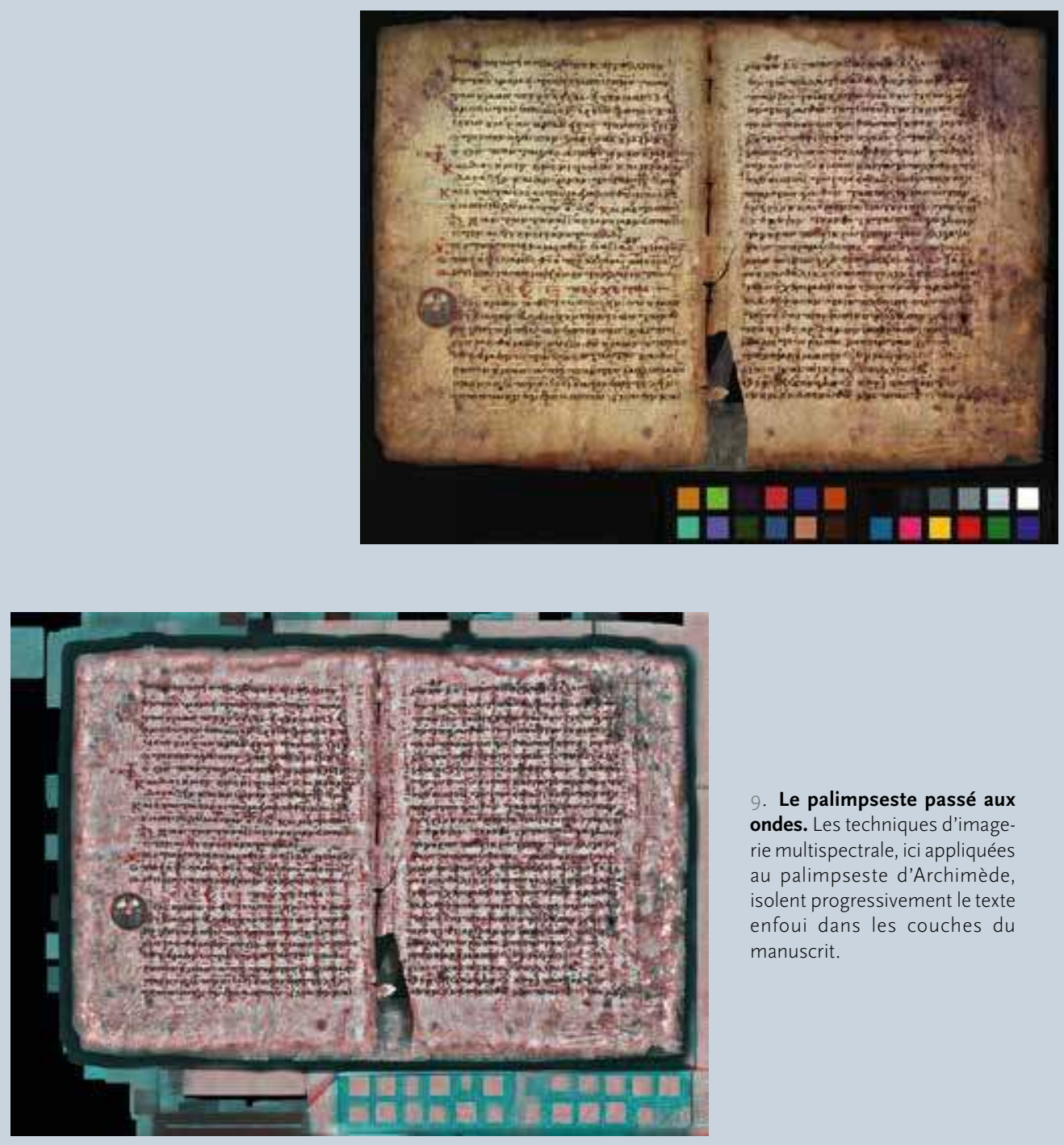

. Le palimpseste passé aux ondes. Les techniques d'imagerie multispectrale, ici appliquées au palimpseste d'Archimède, isolent progressivement le texte enfoui dans les couches du manuscrit.

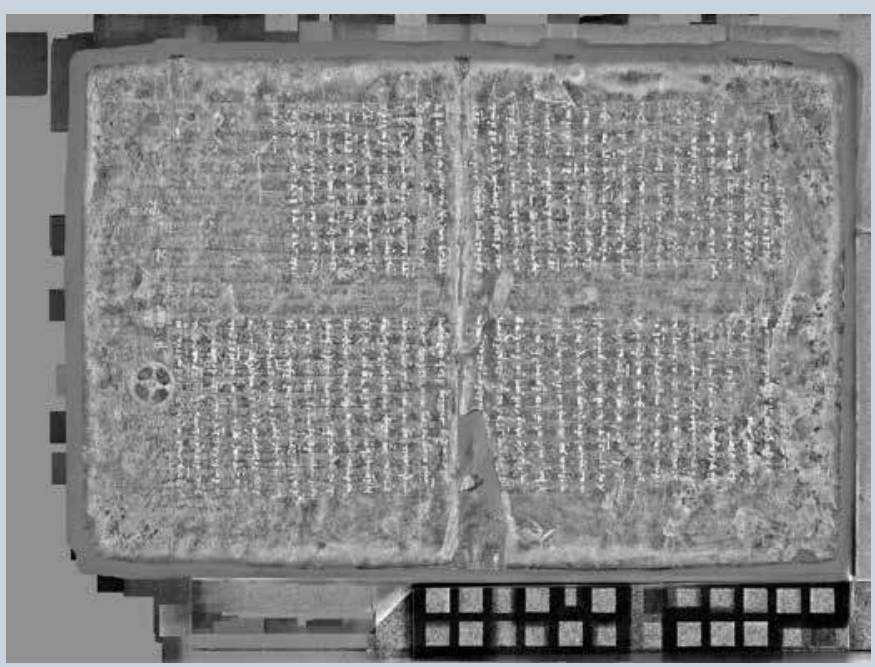


reposent - la passion des estampes et la neutralisation de l'acidité du papier, les techniques d'espionnage et la détection des strates d'écriture - ainsi que d'autres connexions, plus lâches et fragmentées, et les gestes que ces dernières véhiculent fugacement - examiner une moisissure, regarder une larve, palper une feuille.

\section{Notes}

1. Cerquiglini 1989; dans ce sens, les manuscrits arabes n'ont jamais été soumis à une injonction d'originalité de l'ampleur de celle appliquée à partir du XIX ${ }^{\mathrm{e}}$ siècle par la philologie, allemande en particulier, aux manuscrits médiévaux européens; on peut ainsi contraster la «pensée textuaire» promue par la philologie européenne, caractérisée par quête de clôture et de stabilité (ibid.), au texte manuscrit conçu comme, pour reprendre les termes d'un conservateur d'un centre de manuscrits, «ouvert » (fatih), «qui ne se clôt jamais»; voir aussi Jungen 2013.

2. L'immense majorité des manuscrits de langue arabe étant constituée de codex, à savoir des volumes formés de cahiers cousus, j'utiliserai ici le terme pour désigner le livre arabe dans sa qualité de mushaf, «volume»; voir Déroche 2000, Gacek 2012.

3. Soit le tahqiq (littéralement, "authentification»), le travail d'édition critique de textes manuscrits, dont la production se poursuit jusqu'à aujourd'hui à un rythme soutenu.

4. Citons à titre d'exemples les programmes « Mémoires du monde» de l'Unesco au Dar al Kutub, MANUMED financé par l'Union européenne à la Bibliothèque d'Alexandrie.

5. C'est le cas par exemple de la Thesaurus Islamicus Foundation, une organisation internationale qui participe notamment à la restructuration du laboratoire de restauration du Dar al Kutub.

6. La trajectoire de l'expression «manuscrit arabe» communément utilisée dans les cercles érudits et historiens arabophones reste à faire. Remarquons simplement la transformation, avec l'arrivée de l'imprimerie, du «livre» (kitab, renvoyant à l'acte d'écrire) en «manuscrit» (makhtut, de khatta, tracer un trait), qui épouse la relégation progressive du manuscrit dans les collections et bibliothèques spécialisées. Notons par ailleurs également que l'expression est fortement concurrencée par celle de «manuscrit islamique», largement usitée dans le monde anglo-saxon.

7. Plusieurs des collections de manuscrits du Dar al Kutub sont aujourd'hui inscrites au registre «Mémoire du monde » de l'Unesco, qui a également conduit un projet spécifique de valorisation des collections de manuscrits de la bibliothèque. Et si le premier laboratoire de restauration au Dar al Kutub a vu le jour en 1975 avec un soutien logistique soviétique et italien, ce sont aujourd'hui l'agence de coopération espagnole et la Thesaurus Islamicus Foundation, une fondation spécialisée dans l'édition de manuscrits arabes et à vocation tout d'abord académique, qui accompagnent la rénovation des structures de restauration des manuscrits.

8. L'encre dite ferro ou métallo-gallique, largement usitée jusqu'au xix siècle, est constituée à base de sels ferreux qui la rendent, au contraire de l'encre carbone, extrêmement corrosive pour le papier.

9. Le licenciement de Youssef Ziedan fait suite à la parution d'une tribune dans la presse dans laquelle il accuse publiquement le directeur de la Bibliotheca, Ismail Serageldin, de malversations; l'incident intervient peu de mois après un ample mouvement de protestation mené, dans le contexte de la révolution, par les employés contre la gestion de la Bibliotheca et son directeur.

10. «In the Sinai, a Global Team Is Revolutionizing the Preservation of Ancient Manuscripts», Washington Post, 6/9/2012.

11. Voir, par exemple, le cas de la peinture hollandaise dont les œuvres ont été littéralement dé-stabilisées par l'emploi de l'imagerie spectrale (Faries 2003, Hermens \& Fiske 2009). 


\section{I'auteure}

Christine Jungen est anthropologue, chargée de recherche au CNRS, Laboratoire d'ethnologie et de sociologie comparative (LESC). Elle s'intéresse, à partir du Moyen-Orient, au goût pour les vieux papiers, aux environnements matériels de l'histoire, à l'érudition et ses savoir-faire ainsi qu'aux mises au format du passé.

\section{Iconographie}

Image d'ouverture. Quran Carpet. Fragments d'une page enluminée d'un coran de l'époque mamelouke (xive siècle). Source: Bibliothèque du Congrès.

1. \& 2. Bibliothèque nationale et Archives d'Égypte.

3. Source : R. Hooke, Micrographia, Londres, 1665 ; Blue Mold Fig. 1 : 126; Book Worm Fig. 3: 206.

\section{Références}

Blades, W. 1880 The Enemies of Books. Londres.

Bonnardot, A. 1846 Essai sur la restauration des anciennes estampes et des livres rares ou Traité sur les meilleurs procédés à suivre pour réparer, détacher, décolorier et conserver les gravures, dessins et livres: ouvrage spécialement utile aux artistes, aux collectionneurs, aux marchands d'estampes, aux bibliophiles, etc. Paris: Deflorenne Neveu.

Cerquiglini B. 1989 Éloge de la variante. Histoire critique de la philologie. Paris: Seuil.

Click, A. B., Ahmed, S., Hill, J. \& J. D. Martin III 2016 Library and Information Science in the Middle East and North Africa. Berlin/Boston: Walter de Gruyter.

Déroche, F. 2000 Manuel de codicologie des manuscrits en écriture arabe. Paris: Bibliothèque nationale de France.

Déroche, F. \& R. Francis (dir.) 1997 Scribes et manuscrits du Moyen-Orient. Paris: Bibliothèque nationale de France.

Despret, V.2001 Ces émotions qui nous fabriquent: ethnopsychologie de l'authenticité. Paris: Les Empêcheurs de penser en rond / Le Seuil.

Easton, R. L.Jr. \&W. Noel 2004 «The multispectral imaging of the Archimedes palimpsest », Gazette du Livre Médiéval 45(1): 39-49.

Faries, M. 2003 «Technical studies of early netherlandish painting: A critical overview of recent developments», in M. Faries \& R. Spronk (dir.) Recent Developments in the Technical Examination of Early Netherlandish
4. \& 6. (C) C. Jungen.

5. \& 7. (C) Musée des manuscrits, Bibliotheca Alexandrina. 8. Collection de l'American Colony à Jérusalem. Bibliothèque du Congrès.

9. CC BY www.archimedespalimpsest.net.

Painting: Methodology, Limitations and Perspectives. Harvard University Art Museums: Cambridge, MA, en collaboration avec Brepols Publishers: Turnhout: 1-37. Gacek, A. 2012 The Arabic Manuscript Tradition: A Glossary of Technical Terms and Bibliography; Supplement. Leiden: Brill.

Hermens, E. \& T. Diske dir. 2009 Art, Conservation and Authenticities: Material, Concept, Context. Proceedings of the International Conference Held at the University of Glasgow, 12-14 September 2007. Londres: Archetype Publications.

Hooke, R. 1665 Micrographia or Some Physiological Descriptions of Minutes Bodies Made by Magnifying Glasses: With Observations and Inquiries Thereupon. Londres.

Jungen, C. 2013 «Savoirs fragmentés. Des catalogueurs, des bobines de microfilm, et la collecte du "patrimoine arabe et islamique" », Revue d'anthropologie des connaissances 7(4) : 915-939. doi:10.3917/rac.021.0915

Mahdi, M. 1995 «From the manuscript age to the age of printed books» in G. Atiyeh (dir.) The Book in the Islamic World: The Written Word and Communication in the Middle East. Albany: State University of New York Press: 1-15.

Shapin, S. 1998 La révolution scientifique. Paris : Flammarion.

\section{Pour citer l'article}

Jungen, C. 2017 «Le livre au microscope. Petites descriptions écologiques à partir de l'Égypte», Techniques\&Culture 68 «Mondes infimes», p. 196-213. 